\title{
Evaluation of Brain Structure Based on Voxel and Surface Morphology in Adults with MRI Negative Temporal Lobe Epilepsy
}

\section{Fan Yang}

First Affiliated Hospital of Xinjiang Medical University

Hanjiaerbieke Kukun

First Affiliated Hospital of Xinjiang Medical University

Wenxiao Jia

First Affiliated Hospital of Xinjiang Medical University

Shuang Ding

First Affiliated Hospital of Xinjiang Medical University

Wei Zhao

First Affiliated Hospital of Xinjiang Medical University

Yunling Wang ( $\nabla$ 1079806994@qq.com )

First Affiliated Hospital of Xinjiang Medical University

\section{Research Article}

Keywords: Temporal lobe epilepsy, Voxel-based morphometry, surface-based morphometry, structural assessment, epilepsy network

Posted Date: January 10th, 2022

DOI: https://doi.org/10.21203/rs.3.rs-1205693/v1

License: (c) (1) This work is licensed under a Creative Commons Attribution 4.0 International License. Read Full License 


\section{Abstract \\ Background}

MRI-negative TLE (TLE-N) is a manifestation lacks visible MRI findings yet with detectable electrophysiological changes. In this study, differences of gray matter in drug-controlled MRI negative temporal lobe epilepsy (cTLE-N) and drug-resistant MRI negative temporal lobe epilepsy (rTLE-N) patients were calculated and analyzed by voxel-based morphology (VBM) and surface-based morphology (SBM), to discover the brain structural changes of TLE-N patients.

\section{Materials and methods}

Consecutive resident patients with 30 cTLE-N and 21 rTLE-N were recruited into respective groups, and 30 healthy controls' structural MRI (sMRI) data collected as a control group. Open-source software based on VBM and SBM was deployed as gray matter volume (GMV) and cortical thickness (CT) analytic tools.

\section{Results}

VBM analysis showed that GMV of bilateral thalamus and right lingual gyrus of cTLE-N group, and left hippocampus, left fusiform gyrus and left thalamus of rTLE-N group were smaller compared to HC group(FDR corrected, $\mathrm{P}<0.05$ ), while right cerebellum, inferior temporal gyrus, hippocampus, parahippocampal gyrus, amygdala, fusiform gyrus, orbital middle frontal gyrus, and left posterior central gyrus in cTLE-N group, and bilateral cerebellum and middle temporal gyrus, right fusiform gyrus, amygdala, hippocampus, and left middle occipital gyrus of rTLE-N group were greater than HC group(FDR corrected, $\mathrm{P}<0.05)$. SBM analysis showed that CT of the left medial orbitofrontal cortex and lateral occipital cortex in cTLE-N group, and thickness of the left medial orbitofrontal, temporal pole, middle temporal gyrus and right anterior superior cingulate cortex in rTLE-N group were thinner, compared to HC group. Correlation analysis showed that GMV and CT of different structures were correlated with age of onset, disease duration, and MoCA score.

\section{Conclusion}

This study utilized two different SMRI analytic tools and discovered several brain morphological changes in TLE-N. These morphological changes were also correlated with clinical variables. Further study may indicate the potential of these findings on the recognition of the TLE-N epilepsy network.

\section{Background}

Temporal lobe epilepsy (TLE) is the most common type of focal epilepsy in adults, of which one-third of patients are refractory to drug therapy[1]. The rapid development of neuroscience and neuroimaging 
technology provides a good foundation for detection lesions. However, about one-third of TLE patients still show negative results on conventional MRI, and up to $80 \%$ of first-onset epilepsy patients, which affects the establishment of appropriate treatment protocols for this group[2]. TLE is often thought to be associated with hippocampal sclerosis (HS), however there is a significant group of patients with TLE that have no signs of HS or other signs in MRI visual analysis, the so-called MRI negative TLE (TLE-N)[3].

TLE is placed under a high incidence of drug resistance[4]. Compared to TLE patients with HS (TLE-HS), TLE-N patients have a shorter course of disease, a good response to antiepileptic drugs, and less impairment of memory function[2, 5, 6]. With the development of localization techniques, TLE-N patients can also undergo surgical treatment, which often requires an invasive approach to localize the epileptogenic focus preoperatively. A study on the postoperative prognosis of TLE showed that the cure rate of MRI-positive patients was $76 \%$, while that of TLE-N patients was only $38 \%[7]$. Therefore, TLE-N still needed further study.

The cerebral cortex plays a critical role in the occurrence and transmission and is an essential determinant of seizure attacks[8]. Thus, the development of cortical structural exploration is fundamental to understanding the initiation, establishment, and maintenance of networks associated with epileptic seizures. Structural MRI (sMRI), as one of the fundamental methods for epilepsy research and diagnosis, is receiving increasing attention in neuroanatomical morphology and classification. Many studies are evaluating cortical morphometrics in various types of epilepsy. Voxel-based morphometry (VBM) and surface-based morphometry (SBM) are the two main methods of brain morphological analysis to investigate the whole brain grey matter quantitatively.

Although no epileptogenic lesions were seen in the visual assessment of TLE-N by MRI, sMRI covariance analysis based on gray matter volume (GMV) and cortical thickness (CT) can detect structural alterations of the disease by quantitative measures when TLE-N was studied. Based on this approach, sMRI covariance analysis was used to infer the structural networks of different types of TLE. Some researchers have used optimized VBM to evaluate brain damage models between TLE-N and TLE-HS patients, revealing a wide pattern of gray matter atrophy in TLE-HS and TLE-N patients[9, 10]. Mueller et al. employed SBM to confirm the existence of gray matter anomalies in a variety of brain areas in both TLE$\mathrm{N}$ and TLE-HS patients[11]. Regardless of the fact that the gray matter structure of the TLE-N brain has been studied, studies related to CTLE-N and rTLE-N are still limited.

The neuroanatomical features of TLE-N patients have yet to be elucidated, and it is not clear whether these neuroanatomical changes are helpful for clinical diagnosis. In this study, we aimed to explore the alterations in the gray matter structure of CTLE-N and rTLE-N utilizing VBM and SBM analysis. We hypothesized that: (1) there are different patterns of structural gray matter damage in cTLE-N and rTLE-N; (2) there is a correlation between morphological features of abnormal brain regions and clinical variables; and (3) morphological alterations can help us understand the pathological mechanisms of the disease.

\section{Materials And Methods}




\section{Subjects}

Fifty-one patients with TLE and 30 healthy controls $(\mathrm{HC})$ were recruited from the outpatient department of the First Affiliated Hospital of Xinjiang Medical University from February 2020 to May 2021. All the participants were right-handed. Each patient was diagnosed separately by two senior neurologists. This study was approved by the Clinical Trial Ethics Committee of Xinjiang Medical University, and all participants or their family members signed informed consent forms.

\section{Inclusion and exclusion criteria}

Inclusion criteria for the TLE-N group: 1. Patients were diagnosed with TLE based on diagnostic criteria of the International League Against Epilepsy (ILAE)[12]; 2. Refractory epilepsy was diagnosed according to the ILAE 2010 consensus[13]; 3. All patients were monitored by instances of ictal-interictal video EEG using sphenoid sinus electrodes to confirm the source as temporal lobe; 4 . All patients underwent a 3T high resolution MRI with oblique coronal section perpendicular to the long axis of the hippocampus. A comprehensive evaluation by two radiologists confirmed that there were no underlying epileptic structural abnormalities; 5 . Age between 18 and 60 years. The exclusion criteria for the TLE-N group: 1. Long-term history of alcohol or drug abuse; 2 . Severe cognitive impairment; 3 . Previous history of brain trauma, cerebrovascular disease, or brain space-occupying lesions; 4. Poor image quality on MRI examination.

Inclusion criteria for the HC group: 1. No cerebral trauma, history of cerebrovascular disease, or other brain lesions; 2 . No family history of neurological diseases; 3 . Age between 18 and 60 years; 4 . No contraindications to MRI examination. Exclusion criteria for the HC group: 1. Definite personality, mental, emotional disorders, migraine and other diseases that may affect brain functional network connectivity; 2. Severe liver or kidney diseases, diabetes mellitus, and other diseases affecting the nervous system; 3 . Long-term history of alcohol or drug abuse; 4 . Inability to cooperate in completing the examination.

\section{Materials}

General information of the subjects, including name, age, gender, and education level, was recorded. The disease duration, age of onset, EEG, MR, and other examination results and drug treatment for TLE patients were collected. All subjects underwent neuropsychological testing to assess cognitive function prior to MRI, using the Montreal Cognitive Assessment (MoCA) scale for each subject, designed to detect cognitive dysfunction rapidly. It takes approximately $10-15$ minutes to complete, and the total score is 30 , with lower scores indicating severe cognitive impairment ( 1 point is added for $\leq 12$ years of education).

MR acquisition: Visual interpretation of the study results was comprehensively evaluated by two experienced diagnostic radiologists. All subjects underwent image acquisition on a 3.0 T Siemens SKYRA MRI scanner with a 12-channel head coil for scanning. All subjects were first examined with conventional MRI, including axial T1WI, T2WI, and T2-FLAIR scans, to exclude other intracranial lesions. In addition, T1WI acquisition of brain structures using a three-dimensional magnetization prepared rapid gradient echo (MPRAGE) sequence with sagittal scanning, covering the whole brain. The three-dimensional T1- 
weighted MPRAGE sequence with the following parameters: repetition time $=2300 \mathrm{~ms}$, echo time $=2.32$ $\mathrm{ms}$, flip angle $=8^{\circ}$, scanning field of view $=256 \mathrm{~mm} \times 256 \mathrm{~mm}$, matrix size $=256 \times 256$, slice thickness $=$ $0.9 \mathrm{~mm}$, slice spacing $=0$, scan time $=5$ min $21 \mathrm{~s}$.

\section{Methods}

\section{VBM Preprocessing}

VBM analysis was used to explore potential differences in GMV among subjects. Based on SPM12 software and cat12 (http://www.neuro.uni-jena.de/cat/) in MATLAB R2017a, the structural images were firstly aligned to the standard Montreal Neurology Institute (MNI) standard space[14]; Secondly, the normalized T1 images were segmented into gray matter, white matter, and cerebrospinal fluid[15], and whole brain volumes were obtained; Finally, 8mm half-height full-width (FWHM) Gauss check was used to smooth the segmented image.

\section{SBM Preprocessing}

SBM analysis was performed to explore potential differences in CT among subjects. Based on the surf file generated from the above VBM analysis for further analysis, the cortical information processing was performed under CAT12 interface, and the noise of the images was suppressed by resampling \& smoothing to improve the signal-to-noise ratio and reduce the anatomical differences among the images. The size of the smoothing kernel for CT feature processing was $15 \mathrm{~mm}$.

\section{Statistical analysis}

Clinical information: SPSS26.0 software was used for data analysis. Gender was used as a relative number composition ratio (\%), and the $\chi^{2}$ test was used. The measurement data of age, disease duration, age of onset, and MoCA score of the two groups, which conformed to a normal distribution were expressed as means \pm SD. One-way analysis of variance (ANOVA) was used to compare multiple groups, and a two-sample t-test was used to compare between two groups; the measurement data of non-normal distribution were expressed as M (Q1, Q3), and Wilcoxon rank sum test was used. Differences were considered statistically significant at $\mathrm{P}<0.05$.

VBM analysis: To assess the GMV differences among the three groups, a one-way analysis of variance (ANOVA) was performed in a voxel-wise manner in the whole brain $(\mathrm{P}<0.001)$, with whole-brain volume as covariant[16]. Then, the results of ANOVA were used as masks, and post-hoc $T$ tests were used among each group. Voxel-wise false discovery rate (FDR) correction was used for multicomparison correction to control type I error ( $P<0.05$, FDR corrected, cluster size $>100$ voxels). Save the significant regions as masks for further analysis, and mask extraction was done by the toolbox DPABI[17].

SBM analysis: To assess the CT differences among the three groups, a one-way analysis of variance (ANOVA) was performed in a voxel-wise manner in the whole brain $(P<0.001)$. Then, the results of ANOVA were used as masks, and post-hoc $T$ tests were used among each group. Voxel-wise false discovery rate 
(FDR) correction was used for multicomparison correction to control type I error ( $\mathrm{P}<0.05$, FDR corrected). The Desikan-Killiany DK40 Atlas-based CT parameters were extracted for further analysis.

Correlation analysis: The GMV and CT of abnormal brain areas compared with healthy controls were extracted and correlated with clinical variables such as age at onset, duration of disease, and MoCA score, respectively (SPSS26.0), and Pearson correlation analysis was selected if the data of both groups were normally distributed, and Spearman correlation analysis was used if either group did not conform to the normal distribution, with $\mathrm{P}<0.05$ was considered a statistically significant difference.

\section{Results}

\section{General characteristics}

Detailed demographic and clinical data for the CTLE-N, rTLE-N, and HC groups are shown in Table 1. The three groups were well matched for age and gender, with no statistically significant differences $(P>0.05)$. Likewise, the lateralization of TLE did not differ between the cTLE-N and rTLE-N group. RTLE-N patients had a lower age of onset than CTLE-N $(Z=-2.158, P=0.031)$. RTLE-N patients had a longer disease duration than cTLE-N $(Z=-2.211, P=0.027)$. In neurocognitive tests, $r$ TLE-N patients had lower MoCA score than cTLE-N patients $(t=2.787, P=0.009)$. 
Table 1

Clinical Data of the TLE-N Patients and HC

\begin{tabular}{|c|c|c|c|c|}
\hline characteristics & $H C(n=30)$ & cTLE-N(n=30) & rTLE-N(n=21) & $\mathbf{P}$ \\
\hline Age $(y)$ & $38.03 \pm 8.68$ & $34.17 \pm 9.36$ & $32.62 \pm 9.21$ & $0.088^{*}$ \\
\hline Gender (male/female) & $12 / 18$ & $14 / 16$ & $13 / 8$ & $0.299^{\#}$ \\
\hline Lateralization (L/R) & - & $18 / 12$ & $15 / 6$ & $0.553^{\#}$ \\
\hline Age of onset $(y)$ & - & $34(17.25,37)$ & $18(14,20)$ & $0.031^{+}$ \\
\hline Epilepsy durations (y) & - & $5.5(1,20)$ & $13(6.5,18.5)$ & $0.027^{+}$ \\
\hline MoCA Score & - & $25.73 \pm 2.57$ & $23.10 \pm 3.77$ & $0.009^{\sim}$ \\
\hline \multicolumn{5}{|c|}{$\begin{array}{l}\text { Data conforming to a normal distribution are expressed as mean, and non-normal distribution is } \\
\text { expressed as M(Q1, Q3). }\end{array}$} \\
\hline \multicolumn{5}{|c|}{ * The $P$-value was obtained by analysis of variance. } \\
\hline \multicolumn{5}{|c|}{ \# The $P$-value was obtained by a 2-tailed Pearson Chi-square test. } \\
\hline \multicolumn{5}{|c|}{ + The $P$-value was obtained by Wilcoxon rank sum text. } \\
\hline \multicolumn{5}{|c|}{$\sim$ The $P$-value was obtained by 2-sample 2-tailedttest. } \\
\hline
\end{tabular}

\section{Alterations of gray matter volume}

A one-way ANOVA was performed to obtain the difference in GMV among the three groups $(P<0.001)$. Supplementary Figure 1 illustrates the cerebral regions with significant differences among groups, which included the the bilateral hippocampus, middle temporal gyrus and precuneus, right temporal pole (middle temporal gyrus), insula, orbital superior frontal gyrus, orbital middle frontal gyrus, frontal middle gyrus, triangular inferior frontal gyrus, medial and paracingulate gyrus, superior parietal gyrus, supplementary motor area and cerebellum, left middle occipital gyrus, thalamus, amygdala, central precentral gyrus, and postcentral gyrus (Supplementary Table 1).

Post-hoc t-tests were conducted between the three groups to compare the GMV of these regions (Table 2). Compared with the HC group, the cTLE-N group showed reduced GMV in the bilateral thalamus and right lingual gyrus; increased GMV in the right cerebellum, inferior temporal gyrus, hippocampus, parahippocampal gyrus, amygdala, fusiform gyrus and orbital middle frontal gyrus, and left postcentral gyrus region (Figure 1). The rTLE-N group showed decreased GMV in the left hippocampus, fusiform gyrus, and thalamus; increased GMV in the bilateral cerebellum and temporal pole (middle temporal 
gyrus), right fusiform gyrus, amygdala, and hippocampus, and left middle occipital gyrus regions (Figure 2). The rTLE-N group exhibited reduced GMV in the left hippocampus, right insula, and right precuneus and increased GMV in the left middle occipital gyrus compared with the cTLE-N group (Supplementary Figure 2). 
Table 2

Distribution of brain areas with differences in GMV between the two groups $(P<0.05$, FDR corrected)

\begin{tabular}{|c|c|c|c|c|c|c|}
\hline \multirow[t]{2}{*}{ Brain Region(AAL) } & \multirow[t]{2}{*}{ Hemisphere } & \multirow[t]{2}{*}{ Cluster Size } & \multicolumn{3}{|c|}{ MNI(mm) } & \multirow[t]{2}{*}{$t$} \\
\hline & & & $\mathbf{x}$ & $\mathbf{y}$ & $\mathbf{z}$ & \\
\hline \multicolumn{7}{|l|}{ HC>CTLE-N } \\
\hline Lingual & $\mathrm{R}$ & 156 & 15 & -85.5 & -13.5 & 4.03 \\
\hline Thalamus & $\mathrm{L}$ & 845 & -19.5 & -25.5 & 4.5 & 4.72 \\
\hline Thalamus & $\mathrm{R}$ & 165 & 16.5 & -22.5 & 0 & 5.32 \\
\hline \multicolumn{7}{|l|}{$\mathrm{HC}$} \\
\hline Cerebelum_Crus1 & $\mathrm{R}$ & 102 & 42 & -72 & -22.5 & -6.17 \\
\hline Temporal_Inf & $\mathrm{R}$ & 165 & 54 & -15 & -34.5 & -5.95 \\
\hline Hippocampus & $\mathrm{R}$ & 810 & 27 & -4.5 & -25.5 & -6.88 \\
\hline ParaHippocampal & $\mathrm{R}$ & & & & & \\
\hline Amygdala & $\mathrm{R}$ & & & & & \\
\hline Fusiform & $\mathrm{R}$ & 163 & 48 & -37.5 & -28.5 & -6.26 \\
\hline Frontal_Med_Orb & $\mathrm{R}$ & 257 & 9 & 57 & -13.5 & -6.32 \\
\hline Postcentral & $\mathrm{L}$ & 227 & -21 & -40.5 & 66 & -6.34 \\
\hline \multicolumn{7}{|l|}{ HC>rTLE-N } \\
\hline Hippocampus & $\mathrm{L}$ & 328 & -36 & -28.5 & -13.5 & 4.41 \\
\hline Fusiform & $\mathrm{L}$ & 164 & -19.5 & -87 & -9 & 4.51 \\
\hline Thalamus & $\mathrm{L}$ & 537 & 0 & -9 & 13.5 & 4.83 \\
\hline \multicolumn{7}{|l|}{$\mathrm{HC}$} \\
\hline Cerebelum_8 & $\mathrm{L}$ & 128 & -21 & -42 & -49.5 & -5.93 \\
\hline Cerebelum_6 & $\mathrm{R}$ & 582 & 36 & -69 & -21 & -5.38 \\
\hline Fusiform & $\mathrm{R}$ & 229 & 36 & -13.5 & -34.5 & -4.77 \\
\hline Temporal_Pole_Mid & $\mathrm{L}$ & 231 & -52.5 & 12 & -33 & -5.52 \\
\hline Temporal_Pole_Mid & $\mathrm{R}$ & 179 & 49.5 & 21 & -27 & -5.02 \\
\hline Amygdala & $\mathrm{R}$ & 483 & 27 & -4.5 & -13.5 & -4.93 \\
\hline Hippocampus & $\mathrm{R}$ & 103 & & & & \\
\hline
\end{tabular}




\begin{tabular}{|c|c|c|c|c|c|c|}
\hline \multirow[t]{2}{*}{ Brain Region(AAL) } & \multirow[t]{2}{*}{ Hemisphere } & \multirow[t]{2}{*}{ Cluster Size } & \multicolumn{3}{|c|}{ MNI(mm) } & \multirow[t]{2}{*}{$t$} \\
\hline & & & $\mathbf{x}$ & y & $\mathbf{z}$ & \\
\hline Occipital_Mid & L & 334 & -30 & -91.5 & 7.5 & -5.28 \\
\hline \multicolumn{7}{|l|}{ cTLE-N>rTLE-N } \\
\hline Hippocampus & L & 160 & -36 & -22.5 & -18 & 5.71 \\
\hline Insula & $\mathrm{R}$ & 154 & 42 & 16.5 & -7.5 & 5.67 \\
\hline Precuneus & $\mathrm{R}$ & 232 & 10.5 & -69 & 33 & 5.57 \\
\hline \multicolumn{7}{|l|}{ cTLE-N } \\
\hline Occipital_Mid & L & 105 & -28.5 & -94.5 & 7.5 & -4.80 \\
\hline
\end{tabular}

\section{Alterations of Cortical thickness}

A one-way ANOVA was performed to obtain the difference in CT among the three groups $(P<0.001)$. Supplementary Figure 1 illustrates the cerebral regions with significant differences among groups, which included the bilateral parahippocampal gyrus, superior frontal gyrus and precentral gyrus, the left supramarginal gyrus, inferior parietal gyrus, superior temporal gyrus, lateral occipital lobe, inferior middle frontal gyrus, medial orbitofrontal and lateral orbitofrontal, and the right paracentral lobule, superior anterior cingulate gyrus, precuneus, temporal pole, superior parietal gyrus and fusiform gyrus (Supplementary Table 2).

Post-hoc t-tests were conducted between the three groups to compare the CT of these regions. The cTLE$\mathrm{N}$ group exhibited a thinner CT of the left medial orbitofrontal and lateral occipital cortex (Figure 1, Table 3). The rTLE-N group showed thinning of CT in the left medial orbitofrontal, temporal pole, middle temporal gyrus and the upper right anterior cingulate gyrus (Figure 2, Table 4). No brain areas with increased CT were noted in either TLE-N group compared to the control group. The rTLE-N group compared to CTLE-N group showed thinning of CT in the upper anterior cingulate gyrus, precentral gyrus and middle temporal gyrus bilaterally, left superior marginal gyrus, lower middle frontal gyrus, superior temporal gyrus and inferior frontal gyrus orbitally, right postcentral gyrus, paracentral lobule, posterior superior temporal gyrus, superior parietal gyrus, parietal gyrus, inferior parietal gyrus, superior frontal gyrus and precuneus, inferior gyrus, superior frontal gyrus, superior middle frontal gyrus, and precuneus. (Supplementary Figure 2, Table 5). 
Table 3

Brain regions with thinner CT in the cTLE-N group compared with controls (Desikan-Killiany DK40 Atlas)

\begin{tabular}{|c|c|c|c|c|c|c|}
\hline \multirow[t]{2}{*}{ Left Hemisphere } & \multirow[t]{2}{*}{$\mathrm{HC}(\operatorname{Mean}(S D))$} & \multirow[t]{2}{*}{ cTLE-N(Mean(SD)) } & \multicolumn{3}{|c|}{ MNI } & \multirow[t]{2}{*}{$t$} \\
\hline & & & $\mathbf{x}$ & y & $\mathbf{z}$ & \\
\hline medialorbitofrontal & $2.75(0.30)$ & $2.57(0.27)$ & -7 & 52 & -13 & 4.46 \\
\hline lateraloccipital & $2.53(0.33)$ & $2.28(0.14)$ & -40 & -35 & -27 & 3.63 \\
\hline
\end{tabular}

Table 4

Brain regions with thinner $\mathrm{CT}$ in the rTLE-N group compared with controls (Desikan-Killiany DK40 Atlas)

\begin{tabular}{|c|c|c|c|c|c|c|}
\hline \multirow[t]{2}{*}{ Left Hemisphere } & \multirow[t]{2}{*}{ HC(Mean(SD)) } & \multirow[t]{2}{*}{ rTLE-N(Mean(SD)) } & \multicolumn{3}{|c|}{ MNI } & \multirow[t]{2}{*}{$t$} \\
\hline & & & $\mathbf{x}$ & $y$ & $z$ & \\
\hline medialorbitofrontal & $2.82(0.23)$ & $2.56(0.26)$ & 0 & 51 & -16 & 5.36 \\
\hline temporalpole & $3.73(0.27)$ & $3.43(0.25)$ & -28 & 62 & -31 & 3.78 \\
\hline middletemporal & $3.24(0.28)$ & $3.01(0.33)$ & -54 & -15 & -24 & 3.56 \\
\hline \multicolumn{7}{|l|}{ Right Hemisphere } \\
\hline caudalanteriorcingulate & $2.78(0.35)$ & $2.63(0.33)$ & 11 & 62 & 16 & 3.77 \\
\hline
\end{tabular}


Table 5

Brain regions with thinner CT in the rTLE-N group compared with cTLE-N (Desikan-Killiany DK40 Atlas)

\begin{tabular}{|c|c|c|c|c|c|c|}
\hline \multirow[b]{2}{*}{ Left Hemisphere } & \multirow[t]{2}{*}{ cTLE-N(Mean(SD)) } & \multirow[t]{2}{*}{ rTLE-N(Mean(SD)) } & \multicolumn{3}{|c|}{ MNI } & \multirow[t]{2}{*}{$\mathbf{t}$} \\
\hline & & & $\mathbf{x}$ & y & $\mathbf{z}$ & \\
\hline supramarginal & $2.96(0.23)$ & $2.74(0.17)$ & -56 & -57 & 14 & 4.20 \\
\hline rostralanteriorcingulate & $2.84(0.18)$ & $2.61(0.21)$ & -1 & 23 & -4 & 4.92 \\
\hline rostralmiddlefrontal & $2.80(0.18)$ & $2.66(0.13)$ & -39 & 26 & 30 & 4.20 \\
\hline precentral & $2.77(0.32)$ & $2.49(0.33)$ & -31 & -44 & 55 & 3.82 \\
\hline superiortemporal & $3.04(0.22)$ & $2.82(0.25)$ & -61 & -35 & -2 & 4.16 \\
\hline parsorbitalis & $3.01(0.23)$ & $2.84(0.19)$ & -47 & 17 & 6 & 3.62 \\
\hline middletemporal & $3.57(0.29)$ & $3.40(0.29)$ & -57 & 8 & -9 & 3.56 \\
\hline \multicolumn{7}{|l|}{ Right Hemisphere } \\
\hline caudalanteriorcingulate & $2.72(0.20)$ & $2.46(0.26)$ & 7 & 23 & 23 & 5.03 \\
\hline postcentral & $2.45(0.14)$ & $2.26(0.20)$ & 38 & -42 & 35 & 5.27 \\
\hline paracentral & $2.89(0.20)$ & $2.67(0.23)$ & 9 & -36 & 34 & 5.68 \\
\hline bankssts & $2.94(0.14)$ & $2.78(0.21)$ & 51 & -47 & -8 & 4.32 \\
\hline precentral & $3.00(0.20)$ & $2.82(0.22)$ & 62 & -3 & 16 & 4.24 \\
\hline superiorparietal & $2.35(0.18)$ & $2.19(0.16)$ & 14 & -88 & 4 & 5.63 \\
\hline inferiorparietal & $2.75(0.17)$ & $2.56(0.18)$ & 54 & -54 & 1 & 4.67 \\
\hline superiorfrontal & $3.31(0.23)$ & $3.10(0.24)$ & 7 & 7 & 44 & 4.10 \\
\hline caudalmiddlefrontal & $2.88(0.22)$ & $2.75(0.14)$ & 52 & 3 & 35 & 4.09 \\
\hline precuneus & $2.93(0.22)$ & $2.78(0.17)$ & 3 & -58 & 3 & 4.06 \\
\hline middletemporal & $3.16(0.23)$ & $2.93(0.24)$ & 58 & -35 & -14 & 3.95 \\
\hline
\end{tabular}

\section{Correlation analysis}

In CTLE-N group, GMV of left thalamus $\left(r_{s}=-0.399, P=0.029\right)$, right thalamus $\left(r_{s}=-0.404, P=0.027\right)$, right fusiform gyrus $\left(r_{s}=-0.580, P=0.001\right)$, right orbital middle frontal gyrus $\left(r_{s}=-0.643, P=0.001\right)$ were negatively correlated with onset age. The right hippocampal GMV was negatively correlated with the disease duration $\left(r_{s}=-0.454, P=0.012\right)$, and the right hippocampal GMV was positively correlated with MoCA score $\left(r_{p}=0.425, P=0.019\right)$ (Supplementary Table 3$)$. In the rTLE-N group, GMV of the left 
hippocampus $\left(r_{p}=0.603, P=0.004\right)$, left fusiform gyrus $\left(r_{p}=0.634, P=0.002\right)$, left thalamus $\left(r_{p}=0.489\right.$, $P=0.024)$, left temporal pole middle temporal gyrus $\left(r_{s}=0.524, P=0.015\right)$, and left occipital middle gyrus $\left(r_{p}=0.655, P=0.001\right)$ were correlated with MoCA score was positively correlated. Left thalamus GMV was negatively related to disease duration $\left(r_{s}=-0.540, P=0.011\right)$ (Supplementary Table 4).

In the cTLE-N group, CT of the left lateral occipital lobe $\left(r_{s}=-0.477, P=0.008\right)$ and left medial orbitofrontal $\left(r_{s}=-0.425, P=0.019\right)$ was negatively correlated with age at onset. The CT of the left lateral occipital lobe $\left(r_{s}=-0.400, P=0.029\right)$ and left medial orbitofrontal cortex $\left(r_{s}=-0.451, P=0.012\right)$ was negatively correlated with disease duration. The CT of left medial orbitofrontal $\left(r_{p}=0.466, P=0.009\right)$ was positively correlated with MoCA score (Supplementary Table 5). In the rTLE-N group, left medial orbitofrontal $\left(r_{s}=-0.598\right.$, $P=0.004)$ and left middle temporal gyrus $\left(r_{s}=-0.560, P=0.008\right) C T$ was negatively correlated with disease duration. The left medial orbitofrontal $\left(r_{s}=0.411, P=0.024\right)$, left temporal pole $\left(r_{p}=0.503, P=0.020\right)$ and left middle temporal gyrus $\left(r_{p}=0.602, P=0.004\right)$ were positively correlated with MoCA score (Supplementary Table 5).

\section{Discussion}

The purpose of this study was to study the GMV of the TLE-N patients without visible epileptogenic foci by using VBM and SBM morphological analysis methods and to quantitatively detect the regional morphological changes of gray matter in CTLE-N and rTLE-N patients. The morphological differences may help to distinguish rTLE-N patients from cTLE-N patients, to understand the epileptic network of TLE$\mathrm{N}$ patients, and to explain the pathological mechanism and clinical manifestations.

In this study, we distinguished between cTLE-N and rTLE-N patients and found atrophy of hippocampal volume in rTLE-N patients. This suggests that it may be possible to distinguish between CTLE-N and rTLE-N patients using VBM analysis, which detects atrophy of hippocampal volume. Several previous morphological studies showed that hippocampal atrophy was not observed in TLE-N and indicated that TLE-N was a different disease from TLE-HS[18, 19]. It has also been suggested that TLE-N has the same atrophy of hippocampal subregions as TLE-HS, but to a lesser extent, although the total hippocampal volume was not atrophied[20,21]. Our study detected the presence of hippocampal volume atrophy in rTLE-N patients by means of structural MRI covariance analysis based on the GMV, which was consistent with the pathological conclusion that TLE-N has relatively mild hippocampal neuronal cell loss[22]. The observation may support the hypothesis that rTLE-N may be a mild manifestation of TLE-HS that has not yet shown changes in hippocampal volume observable to the naked eye.

Another significant finding was atrophy of thalamic GMV in both groups of TLE-N groups. The thalamus plays an essential role in the regulation, diffusion and termination of epileptic seizures[23-25], which may be due to the epileptogenic network connecting the anterior thalamus and the medial temporal lobe. These results further support the idea that the thalamus is an essential part of the TLE-N epileptogenic network, and the GMV of the thalamus plays a crucial pathophysiological role in the disease. A clinical 
study found that deep brain stimulation surgery in the subthalamic nucleus was effective in controlling seizures [26], which may become a routine and effective treatment for rTLE-N patients.

It has been suggested that extensive GMV atrophy in TLE-N patients [18]. This does not appear to be the case. In our study, one unanticipated result was increased GMV in the limbic system (right hippocampus, right amygdala), temporal lobe structures (inferior temporal gyrus, temporal pole) and fusiform gyrus in both CTLE-N and rTLE-N patients. Clinical studies have shown that patients' brain structures, when affected by epileptic discharges, initially exhibit brain cell death and later a large number of glial cells proliferate. These cells are involved in the repair and regeneration of the nervous system, thus causing a larger volume in the relevant brain regions[27]. The results of this study may explain this view from the perspective of morphological structure, that is, the hippocampus and peripheral regions, as areas affected by long-lasting epileptic discharge, may undergo volume changes as described above. We also found that amygdala enlargement was in both groups. In recent years, amygdala enlargement (AE)associated TLE has been considered a subtype of TLE-N[28]. Sone et al found that the amygdala volume increased in TLE-AE patients compared with the control group, and the hippocampal volume also increased slightly in TLE-AE patients[29]. We found an increase in the right amygdala and hippocampus volume in both cTLE-N and rTLE-N groups, which were different from TLE-HS. In rTLE-N patients, the GMV of the left hippocampus and fusiform gyrus was atrophic, and the GMV of the right hippocampus and the fusiform gyrus was increased, which were represented by atrophy on one side and compensation on the other side. In CTLE-N patients, the GMV of the right hippocampus and the fusiform gyrus only increased. According to these data, we can infer that cTLE-N may be a pre-expression of rTLE-N. The morphology of the similar structures described above may suggest that cTLE-N and rTLE-N have similar pathological mechanisms from the morphological perspective. Compared with cTLE-N, rTLE-N patients showed atrophy of GMV in the left hippocampus, right insula, and right precuneus, suggesting that there were some differences between rTLE-N and CTLE-N epileptogenic network, with severe damage to rTLE-N in the appealed structures.

Among the subjects included in this study, rTLE-N patients were found to have an earlier age of onset and a longer disease duration than CTLE-N patients. Structures such as the hippocampus are in growth and maturity during infancy. They are susceptible to external influences, and that various injuries during infancy (such as birth injury, trauma, thermal convulsion, etc.) lead to hypoxia of brain tissue, degeneration, necrosis, loss and reactive gliosis of hippocampus neurons, which gradually evolve into HS[30]. The rTLE-N patients included in our study were consistent with this clinical change, and the atrophy of the left hippocampus was negatively correlated with the disease duration and positively correlated with MoCA score. Long-term atrophy of the hippocampus could affect cognitive function. Meanwhile, the GMV of the left fusiform gyrus, thalamus, temporal pole middle temporal gyrus and middle occipital gyrus was correlated with MoCA score, and only the right hippocampal volume correlated with MoCA score in CTLE-N patients. The fusiform gyrus is located in the superior part of the parahippocamal gyrus and lingual gyrus, and in the lower part of the inferior temporal gyrus. The fusiform gyrus is associated with neural pathways related to visual recognition[31, 32]. The middle occipital gyrus plays a significant role in vision and language[33]. The temporal lobe is generally related 
to language, hearing, memory, emotion and other functions, and its main function is processing language[34]. These structural abnormalities are correlated with clinical variables and can be utilized to explain clinical phenomena. But further explanation and verification need to be combined with functional imaging studies.

When the CT of subjects was studied based on SBM, we found that the CT of some brain regions was thinned in cTLE-N and rTLE-N group and no thickened area. The cTLE-N group showed thinning of the left medial orbitofrontal and lateral occipital cortex. In contrast, the rTLE-N group showed CT thinning of the left medial orbitofrontal, temporal pole, middle temporal gyrus and the right anterior superior cingulate cortex. Moreover, combined with the results of VBM analysis, we found that CT atrophy of temporal lobe structures was also decreased on the ipsilateral side of the hippocampal atrophy. It has been confirmed that chronic epileptic discharge in the structure of the medial temporal lobe leads to structural changes. Thinning of medial orbitofrontal cortex was found in both cTLE-N and rTLE-N groups. The medial orbitofrontal cortex is located in the prefrontal lobe, which is closely connected between the prefrontal and hippocampus and plays an essential role in higher cognitive functions[35, 36]. Studies have shown that through the connection between the prefrontal lobe and hippocampus, epileptic discharges propagate to the prefrontal region, leading to its structural alterations[37], and we discovered a negative correlation between alterations in medial orbitofrontal CT and disease duration and a positive correlation with MoCA scores in both groups of patients, and the abnormalities in frontal lobe structure may be due to long-term epileptic discharges in the hippocampus and consequently affect the cognitive function of the patients. Hence, it could conceivably be hypothesized that the frontal lobe structure had been involved before the hippocampal atrophy in the cTLE-N group, and thus CTLE-N may be a prodromal manifestation of rTLE-N.

There are several limitations of this study. First, the number of patients enrolled in the two case groups was small. A larger sample is needed to validate our findings. Second, the potential pathological causes associated with morphological alterations in different brain regions still need further in-depth analysis in combination with clinical or relevant functional imaging, etc. Third, the lack of consensus on the ideal setting of parameters in neuroimaging by the structural image-based morphological analysis approach can lead to discrepancies among the existing relevant studies. Despite these limitations, our findings may also contribute to the use of VBM and SBM to understand the epileptic network of TLE-N.

\section{Conclusions}

It was shown that the presence of regional gray matter morphological alterations in cTLE-N and rTLE-N patients could be quantified by both VBM and SBM morphological analysis. These morphological changes were correlated with clinical variables. There were similar morphological changes between cTLE$\mathrm{N}$ and rTLE-N, and we speculated that the two might also have similar pathological mechanisms. CTLE-N might be a precursor of rTLE-N and rTLE-N might be a mild TLE-HS. Due to the complexity of epilepsy networks, more studies are needed to verify this conclusion. 


\section{Declarations}

\section{Availability of data and material}

The authors declare that all data supporting the findings of this study are available within the paper and its source data for the figures in this study are available from the authors upon request.

\section{Acknowledgements}

Not applicable.

\section{Funding}

This work was supported by Regional Collaborative Innovation special fund of Xinjiang Uygur Autonomous Region (Grant numbers: 2020E0275).

\section{Authors Information}

Department of Radiology, The First Affiliated Hospital, Xinjiang Medical University

137 Liyushan South Road, Xincheng District, Urumqi, Xinjiang, China

\section{Authors' contributions}

Fan Yang and Hanjiaerbieke Kukun contributed equally to this manuscript.

Wenxiao Jia proposed the research idea. Wei Zhao and Hanjiaerbieke Kukun carried out image acquisition. Fan Yang carried out experiments, collected structures and drafted papers. Shuang Ding and Yunling Wang proofread the article. Both authors read and approved the final manuscript.

\section{Ethics approval and consent to participate}

This study was approved by the Clinical Trial Ethics Committee of Xinjiang Medical University, and all participants or their family members signed informed consent forms. All methods were carried out in accordance with relevant guidelines and regulations.

\section{Consent for publication}

Not applicable.

\section{Competing interests}

The authors declare that they have no competing interests.

\section{References}


1. Engel J: Mesial temporal lobe epilepsy: what have we learned? The Neuroscientist: a review journal bringing neurobiology, neurology and psychiatry 2001, 7:340-352.

2. Muhlhofer W, Tan Y, Mueller S, Knowlton R: MRI-negative temporal lobe epilepsy-What do we know? Epilepsia 2017, 58:727-742.

3. Bower S, Vogrin S, Morris K, Cox I, Murphy M, Kilpatrick C, Cook M: Amygdala volumetry in "imagingnegative" temporal lobe epilepsy. Journal of neurology, neurosurgery, and psychiatry 2003, 74:12451249.

4. Asadi-Pooya A, Stewart G, Abrams D, Sharan A: Prevalence and Incidence of Drug-Resistant Mesial Temporal Lobe Epilepsy in the United States. World neurosurgery 2017, 99:662-666.

5. Reyes A, Thesen T, Wang X, Hahn D, Yoo D, Kuzniecky R, Devinsky O, Blackmon K: Resting-state functional MRI distinguishes temporal lobe epilepsy subtypes. Epilepsia 2016, 57:1475-1484.

6. Kim J, Kim S, Lim S, Kim W, Shon Y: Clinical characteristics of patients with benign nonlesional temporal lobe epilepsy. Neuropsychiatric disease and treatment 2016, 12:1887-1891.

7. Bien C, Szinay M, Wagner J, Clusmann H, Becker A, Urbach H: Characteristics and surgical outcomes of patients with refractory magnetic resonance imaging-negative epilepsies. Archives of neurology 2009, 66:1491-1499.

8. Paul A, Fathima K, Nair S: In situlntra Nasal Gelling System of Lamotrigine Using lon Activated Mucoadhesive Polymer. The open medicinal chemistry journal 2017, 11:222-244.

9. Bonilha L, Halford J: Network atrophy in temporal lobe epilepsy: a voxel-based morphometry study. Neurology 2009, 72:2052; author reply 2052.

10. Scanlon C, Mueller S, Cheong I, Hartig M, Weiner M, Laxer K: Grey and white matter abnormalities in temporal lobe epilepsy with and without mesial temporal sclerosis. Journal of neurology 2013, 260:2320-2329.

11. Mueller S, Laxer K, Barakos J, Cheong I, Garcia P, Weiner M: Widespread neocortical abnormalities in temporal lobe epilepsy with and without mesial sclerosis. Neurolmage 2009, 46:353-359.

12. Berg A, Berkovic S, Brodie M, Buchhalter J, Cross J, van Emde Boas W, Engel J, French J, Glauser T, Mathern G, et al: Revised terminology and concepts for organization of seizures and epilepsies: report of the ILAE Commission on Classification and Terminology, 2005-2009. Epilepsia 2010, 51:676-685.

13. Kwan P, Arzimanoglou A, Berg A, Brodie M, Allen Hauser W, Mathern G, Moshé S, Perucca E, Wiebe S, French J: Definition of drug resistant epilepsy: consensus proposal by the ad hoc Task Force of the ILAE Commission on Therapeutic Strategies. Epilepsia 2010, 51:1069-1077.

14. Klein A, Ghosh S, Parsey R: An evaluation of volume- and surface-based nonlinear registration of human brain MRI data. Neuroimage 2009, 47:S123-S123.

15. Ashburner J, Friston KJ: Unified segmentation. Neuroimage 2005, 26:839-851.

16. Pell GS, Briellmann RS, Chan CH, Pardoe H, Abbott DF, Jackson GD: Selection of the control group for VBM analysis: influence of covariates, matching and sample size. Neuroimage 2008, 41:1324-1335. 
17. Yan CG, Wang XD, Zuo XN, Zang YF: DPABI: Data Processing \& Analysis for (Resting-State) Brain Imaging. Neuroinformatics 2016, 14:339-351.

18. Scanlon C, Mueller SG, Cheong I, Hartig M, Weiner MW, Laxer KD: Grey and white matter abnormalities in temporal lobe epilepsy with and without mesial temporal sclerosis. Journal of Neurology 2013, 260:2320-2329.

19. Mueller SG, Laxer KD, Cashdollar N, Buckley S, Paul C, Weiner MW: Voxel-based Optimized Morphometry (VBM) of Gray and White Matter in Temporal Lobe Epilepsy (TLE) with and without Mesial Temporal Sclerosis. Epilepsia 2006, 47.

20. Mumoli L, Labate A, Vasta R, Gambardella A: Letter: Beyond and within CA1 subfield in magnetic resonance imaging negative temporal lobe epilepsy. Epilepsia 2015, 56:1471-1471.

21. Bernhardt BC, Kim H, Bernasconi N: Patterns of subregional disease progression in temporal lobe epilepsy. Neurology 2013, 81.

22. Yang PF, Pei JS, Zhang HJ, Lin Q, Mei Z, Zhong ZH, Tian J, Jia YZ, Chen ZQ, Zheng ZY: Long-term epilepsy surgery outcomes in patients with PET-positive, MRI-negative temporal lobe epilepsy. Epilepsy \& Behavior E \& B 2014, 41:91-97.

23. Elisa E, Christian B, Francesca B, Romain C, Bruno C, Jean R, Fabrice B: Does the Thalamo-Cortical Synchrony Play a Role in Seizure Termination? Frontiers in Neurology 2015, 6.

24. Dreifuss S, Vingerhoets F, Lazeyras F, Andino SG, Spinelli L, De Lavelle J, Seeck M: Volumetric measurements of subcortical nuclei in patients with temporal lobe epilepsy. Neurology 2001, 57:1636-1641.

25. Bertram EH, Mangan PS, Zhang DX, Scott CA, Williamson JM: The midline thalamus: alterations and a potential role in limbic epilepsy. Epilepsia 2010, 42:967-978.

26. Electrical stimulation of the anterior nucleus of thalamus for treatment of refractory epilepsy. Epilepsia 2010, 51.

27. Newman E: New roles for astrocytes: regulation of synaptic function. TRENDS IN NEUROSCIENCES 2003.

28. Lv RJ, Sun ZR, Cui T, Guan HZ, Ren HT, Shao XQ: Temporal lobe epilepsy with amygdala enlargement: a subtype of temporal lobe epilepsy. Bmc Neurology 2014, 14:1-8.

29. Iman B, Daichi S, arnaz FF, Norihide M, Hiroshi M: Gray Matter and White Matter Abnormalities in Temporal Lobe Epilepsy Patients with and without Hippocampal Sclerosis. Frontiers in Neurology 2018, 9:107-.

30. Pierre G: Experiential phenomena of temporal lobe epilepsy. Facts and hypotheses. Brain A Journal of Neurology: 1673.

31. Zhang X, Japee S, Safiullah Z, Mlynaryk N, Ungerleider LG: A Normalization Framework for Emotional Attention. PLoS Biology 2016, 14:e1002578.

32. Weiner, Kevin, S., Zilles, Karl: The anatomical and functional specialization of the fusiform gyrus. Neuropsychologia 2016. 
33. Deng X, Liu Z, Kang Q, Lu L, Zhu Y, Xu R: Cortical Structural Connectivity Alterations and Potential Pathogenesis in Mid-Stage Sporadic Parkinson's Disease. Frontiers in aging neuroscience 2021, 13:650371.

34. Anderson J, Treiman S, Ferguson M, Nielsen J, Edgin J, Dai L, Gerig G, Korenberg J: Violence: heightened brain attentional network response is selectively muted in Down syndrome. Journal of neurodevelopmental disorders 2015, 7:15.

35. Wood JN, Grafman J: Human prefrontal cortex: processing and representational perspectives. Nature Reiview Neuroscience 2003, 4:139-147.

36. Lieb JP, Da Sheiff RM, Engel J: Role of the Frontal Lobes in the Propagation of Mesial Temporal Lobe Seizures. Epilepsia 1991, 32:822-837.

37. Schacher M, Winkler R, Grunwald T, Kraemer G, Kurthen M, Reed V, Jokeit H: Mesial Temporal Lobe Epilepsy Impairs Advanced Social Cognition. Epilepsia 2010, 47:2141-2146.

\section{Figures}

\section{Figure 1}

VBM and SBM analysis between cTLE-N group and HC group

(In the VBM results, blue represents the decreased GMV of cTLE-N group, and yellow represents the increased GMV of cTLE-N group; In SBM results, color labeling indicated cortical thinning areas in cTLE-N group)

\section{Figure 2}

VBM and SBM analysis between rTLE-N group and HC group

(In the VBM results, blue represents the decreased GMV of rTLE-N group, and yellow represents the increased GMV of rTLE-N group; In SBM results, color labeling indicated cortical thinning areas in rTLE-N group)

\section{Supplementary Files}

This is a list of supplementary files associated with this preprint. Click to download.

- supplementarypictureandtable.zip 